\title{
Decomposition of the static potential in the Maximal Abelian gauge
}

\author{
Vitaly Bornyakov ${ }^{1,2,3, *}$, Vladimir Goy ${ }^{2,4}$, Ilya $\mathrm{Kudrov}^{3}$, and Roman Rogalyov ${ }^{1}$ \\ ${ }^{1}$ Institute for High Energy Physics NRC Kurchatov Institute, 142281 Protvino, Russia \\ ${ }^{2}$ Pacific Quantum Center, Far Eastern Federal University, 690950 Vladivostok, Russia \\ ${ }^{3}$ Institute of Theoretical and Experimental Physics NRC Kurchatov Institute, 117218 Moscow, Russia \\ ${ }^{4}$ Institut Denis Poisson CNRS/UMR 7013, Université de Tours, 37200 France
}

\begin{abstract}
Decomposition of SU(2) gauge field into monopole and monopoleless components is studied in $S U(2)$ gluodynamics and in $Q C_{2} D$ with zero and nonzero quark chemical potential after fixing MA gauge. For both components we calculate respective static potential and compare their sum with the nonabelian static potential. We demonstrate good agreement in the confinement phase and discuss the implications of our results.
\end{abstract}

\section{Introduction}

We study decomposition of the nonabelian gauge field in the Maximal Abelian gauge (MAG) $[1,2]$ into the sum of the monopole component and the monopoleless component. We use the lattice gauge theory with gauge group $S U(2)$ for this purpose. In terms of the lattice gauge field $U_{\mu}(x)$ the decomposition has the following form

$$
U_{\mu}(x)=U_{\mu}^{\bmod }(x) u_{\mu}^{m o n}(x)
$$

where $u_{\mu}^{\text {mon }}(x)$ is the monopole component and $U_{\mu}^{\text {mod }}(x)$ is the monopoleless component called below also a modified gauge field.

The Abelian dominance for the string tension after fixing MAG [1, 2] is known [3-7] since long (for a review see e.g. [8, 9]). Moreover, it was found $[5,10,11]$ that the properly determined monopole component of the gauge field produces the string tension close to its exact value in agreement with conjecture that the monopole degrees of freedom are responsible for confinement $[12,13]$.

In Refs. $[14,15]$ it was shown that the topological charge, chiral condensate and effects of chiral symmetry breaking in quenched light hadron spectrum disappear after removal of the monopole contribution from the relevant operators. Similar computations were made within the scope of the $Z_{2}$ projection studies [16]. In particular it was shown that after removal of $\mathrm{P}$-vortices the confinement property disappear. We do a similar removal with monopoles. We consider the following types of the static potential: $V_{\bmod }(r)$ obtained from the Wilson loops of the modified gauge field $U_{\mu}^{\text {mod }}(x), V_{\text {mon }}(r)$ obtained from the Wilson loops of the monopole gauge field $u_{\mu}^{m o n}(x)$ and the sum of these two static potentials.

\footnotetext{
*e-mail: vitaly.bornyakov@ihep.ru
} 
It was demonstrated in [17] for one value of the lattice spacing that $V_{\text {mod }}(r)$ could be well fitted by purely Coulomb fit function and the sum $V_{\text {mod }}(r)+V_{\text {mon }}(r)$ was a good approximation of the original nonabelian static potential, $V(r)$, at all distances.

Here we study this phenomenon at three lattice spacings using the Wilson lattice gauge field action and thus we can make conclusions about the continuum limit. We also present the results for one lattice spacing obtained with the improved lattice field action thus checking the universality. Furthermore, we present results for the $S U(2)$ theory with dynamical quarks, i.e. for $\mathrm{QC}_{2} \mathrm{D}$. These results were partially presented in [18].

\section{Details of simulations}

We study the $S U(2)$ lattice gauge theory. To fix MAG we used the simulated annealing algorithm [5] with one gauge copy per configuration. Usually after fixing MAG the following decomposition of the nonabelian lattice gauge field $U_{\mu}(x)$ is made

$$
U_{\mu}(x)=C_{\mu}(x) u_{\mu}(x)
$$

where $u_{\mu}(x)$ is the Abelian field and $C_{\mu}(x)$ is the nonabelian coset field. The Abelian gauge field $u_{\mu}(x)$ is further decomposed [19] into the monopole (singular) part $u_{\mu}^{\text {mon }}(x)$ and the photon (regular) part $u_{\mu}^{p h}(x)$ :

$$
u_{\mu}(x)=u_{\mu}^{m o n}(x) u_{\mu}^{p h}(x) .
$$

Then it follows from eq. (1) that

$$
U_{\mu}^{m o d}(x)=C_{\mu}(x) u_{\mu}^{p h}(x)
$$

Note that $u^{p h}(x)$ is the Abelian projection of $U_{\mu}^{\bmod }(x)$ and involves no monopoles.

We need to compute the usual Wilson loops

$$
W(C)=\frac{1}{2} \operatorname{Tr} \mathcal{W}(C), \quad \mathcal{W}(C)=\left(\prod_{l \in C} U(l)\right),
$$

the monopole Wilson loops

$$
W_{\text {mon }}(C)=\frac{1}{2} \operatorname{Tr}\left(\prod_{l \in C} u^{m o n}(l)\right),
$$

and the nonabelian Wilson loops with removed monopole contribution

$$
W_{\text {mod }}(C)=\frac{1}{2} \operatorname{Tr} W_{\text {mod }}(C), \quad W_{\text {mod }}(C)=\left(\prod_{l \in C} U^{\text {mod }}(l)\right),
$$

It is known that MAG fixing leaves $U(1)$ gauge symmetry unbroken. The monopole Wilson loop $W_{\text {mon }}(C)$ is invariant under respective residual gauge transformations. This is not true for $W_{\text {mod }}(C)$ [17]. Thus we need to fix the Landau $U(1)$ gauge using the gauge condition

$$
\max _{\omega} \sum_{x, \mu} \operatorname{Re}\left(\omega(x) u_{\mu}(x) \omega^{\dagger}(x+\mu)\right)
$$

where $\omega \in U(1)$ is the gauge transformation. To imrove the noise to signal ratio for the static potential we used the APE smearing [20] in computations of the Wilson loops. 


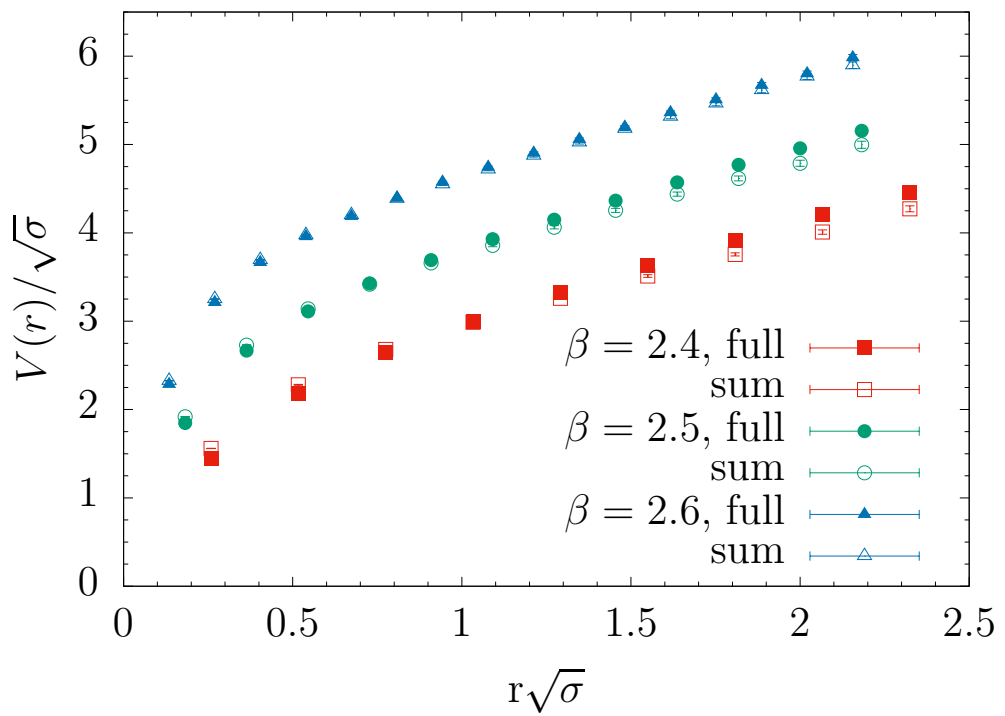

Figure 1. Comparison of the nonabelian static potential $V(r)$ (filled symbols) with the sum $V_{\text {mon }}(r)+$ $V_{\text {mod }}(r)$ (empty symbols) for Wilson action at $\beta=2.4$ (squares), $\beta=2.5$ (circles), $\beta=2.6$ (triangles).

We generated 100 statistically independent gauge field configurations with the Wilson lattice action at $\beta=2.4,2.5$ on $24^{4}$ lattices and at $\beta=2.6$ on $32^{4}$ lattices. As a check of universality the computations were done with the tadpole improved action at $\beta=3.4$ on $24^{4}$ lattices. Additionally we present our results obtained in $\mathrm{QC}_{2} \mathrm{D}$ on $32^{4}$ lattice at zero and nonzero quark chemical potential [21].

It is worth to note that another decomposition, namely eq. (2) was investigated in Ref. [7] in case if $S U(3)$ gluodynamics. Good agreement between the static potenial $V(r)$ and the sum $V_{a b e l}(r)+V_{\text {off }}(r)$ was found. We believe that this decomposition also deserves further study.

\section{Results}

In Figure 1 we show the usual nonabelian static potential $V(r)$ denoted as 'full' and compare it with the sum $V_{\text {sum }}(r)=V_{\text {mon }}(r)+V_{\text {mod }}(r)$. One can see that approximate equality

$$
V(r) \approx V_{\text {sum }}(r)
$$

is satisfied for all three lattice spacings and the approximation improves toward the continuum limit. To give further support to this statement we plot in Figure 2 the relative deviation $\Delta(r)$ defined as

$$
\Delta(r)=\frac{V(r)-V_{\text {sum }}(r)}{V(r)} .
$$

It is clear that $\Delta(r)$ decreases with decreasing lattice spacing.

We also studied the question of universality of the decomposition for the static potential eq. (9). The simulations were made with the tadpole improved action at $\beta=3.4$ with lattice spacing approximately equal to that of the Wilson action at $\beta=2.5$. The results of our computations are presented in the Figure 3. One can see that agreement between $V(r)$ and $V_{\text {sum }}(r)$ is nearly as good as in Figure 1 for $\beta=2.5$. 


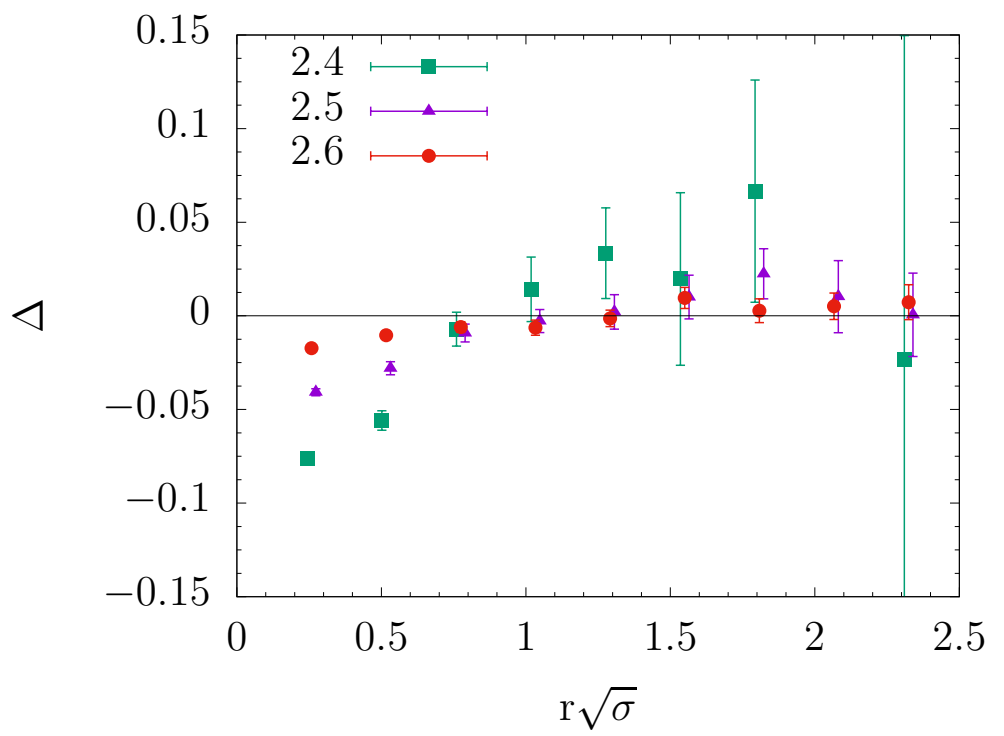

Figure 2. The relative deviation $\Delta(r)$ vs. distance $r$ for three values of the lattice spacing.

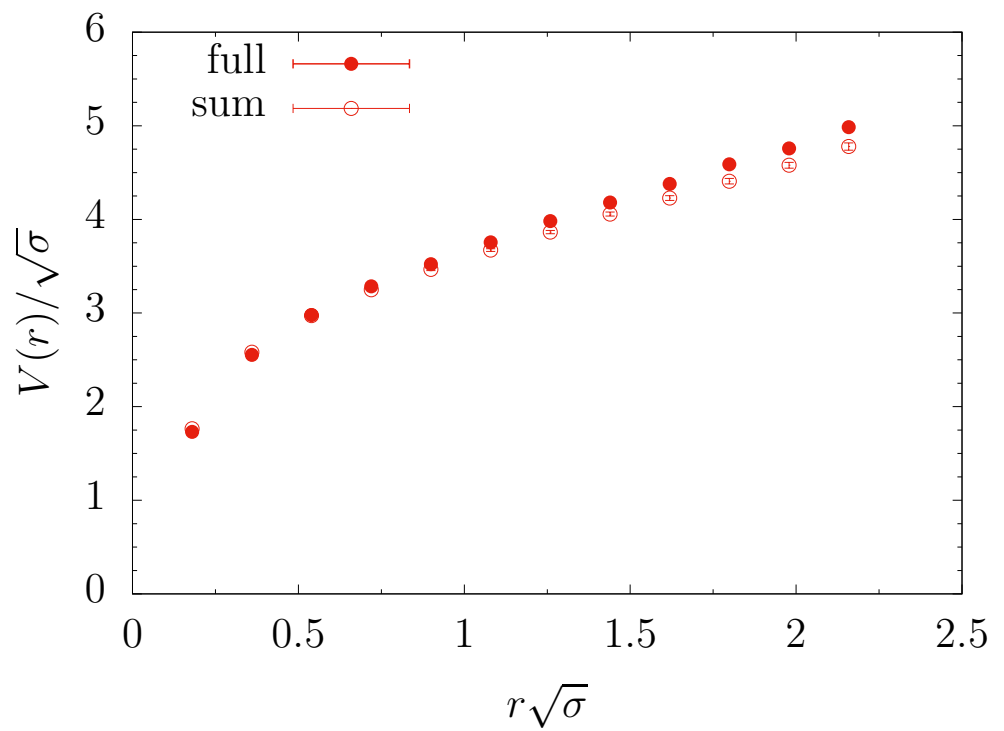

Figure 3. Comparison of the nonabelian static potential $V(r)$ (filled symbols) with the sum of the monopole and modified potentials $V_{\text {sum }}(r)$ (empty symbols) for improved action at $\beta=3.4$.

Furthermore, we completed the same computations in $\mathrm{QC}_{2} \mathrm{D}$ on $32^{4}$ lattices with small lattice spacing at zero and nonzero quark chemical potential $\mu_{q}$ (for details of simulations in $\mathrm{QC}_{2} \mathrm{D}$ see, e.g. [21]). The results of these computations are presented in Figure 4 for $\mu_{q}=0$ and in Figure 5 for $a \mu_{q}=0.19$. It can be seen clearly that approximate decomposition (9) is fulfilled with rather high both at zero and nonzero $\mu_{q}$. 


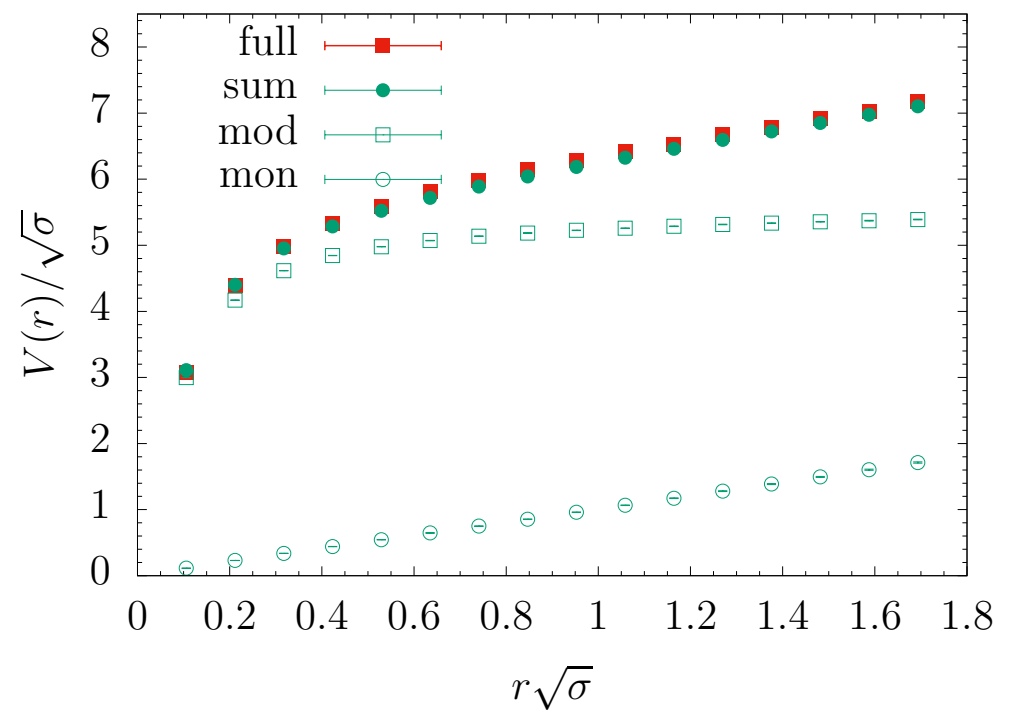

Figure 4. The nonabelian static potential $V(r)$ (filled squares) is compared with the sum of the monopole and modified potentials $V_{\text {sum }}(r)$ (filled circles) for $\mathrm{QC}_{2} \mathrm{D}$ at zero chemical potential. The potentials $V_{\text {mon }}(r)$ (empty circles) and $V_{\text {mod }}(r)$ (empty squares) are also shown.

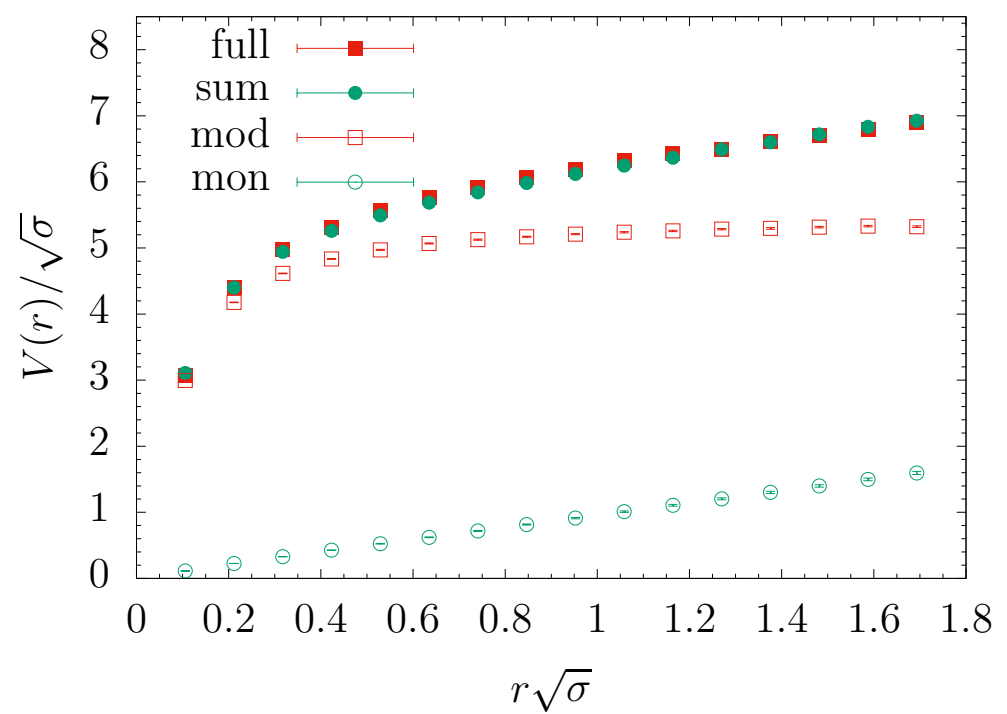

Figure 5. The nonabelian static potential $V(r)$ (filled squares) is compared with the sum of the monopole and modified potentials $V_{\text {sum }}(r)$ (filled circles) for $\mathrm{QC}_{2} \mathrm{D}$ at nonzero chemical potential $a \mu_{q}=0.19$. The potentials $V_{\text {mon }}(r)$ (empty circles) and $V_{\text {mod }}(r)$ (empty squares) are also shown.

\section{Conclusions}

We studied the decomposition of the static potential into the linear term produced by the monopole (Abelian) gauge field $U_{m o n}(x)$ and the Coulomb term produced by the monopoleless nonabelian gauge field $U_{\text {mod }}(x)$. In case of the Wilson action we presented results (Figures 1 and 2) for three lattice spacings demonstrated that the agreement becomes better with 
decreasing lattice spacing. Thus our results imply that the relation (1) becomes exact in the continuum limit. Further work is needed to provide more evidence for this conclusion. Next, we demonstrated that the decomposition (1) is valid also in the case of improved lattice action (see Figure 3). Furthermore, we found that it works also in $\mathrm{QC}_{2} \mathrm{D}$ for both zero and nonzero quark chemical potential. Let us note that in Ref. [18] we also presented results for the adjoint static potential and found that the decomposition (1) works quite well in this case although not as well as in the case of the fundamental representation. It is of course interesting to verify how decomposition (1) works for other observables, e.g. action density and energy density of the flux tube. Also the decomposition should be checked in the case of SU(3) gauge group.

One can draw the following conclusions from the decomposition (1). The monopole part of the gauge field $U_{m o n}(x)$ is responsible for the classical part of the hadronic string energy while the monopoleless part $U_{\text {mod }}(x)$ produces the fluctuating part of that energy, i.e. while at small distances $U_{\text {mod }}(x)$ should reproduce the perturbative results at large distances it contributes to the nonperturbative physics.

Acknowledgments. Computer simulations were performed on the Central Linux Cluster of the NRC "Kurchatov Institute" - IHEP (Protvino) and Linux Cluster of the NRC "Kurchatov Institute" - ITEP (Moscow). This work was supported by the Russian Foundation for Basic Research, grant no.20-02-00737 A. The authors are grateful to G. Schierholz, T. Suzuki, S. Syritsyn, V. Braguta, A. Nikolaev for participation at the early stages of this work and for useful discussions.

\section{References}

[1] A. S. Kronfeld, M. L. Laursen, G. Schierholz and U. J. Wiese, Phys. Lett. B 198, 516 (1987).

[2] G. 't Hooft, Nucl. Phys. B 190 (1981), 455-478 doi:10.1016/0550-3213(81)90442-9

[3] T. Suzuki and I. Yotsuyanagi, Phys. Rev. D 42 (1990) 4257.

[4] S. Hioki, S. Kitahara, S. Kiura, Y. Matsubara, O. Miyamura, S. Ohno and T. Suzuki, Phys. Lett. B 272, 326 (1991) [Erratum-ibid. B 281, 416 (1992)].

[5] G. S. Bali, V. Bornyakov, M. Muller-Preussker and K. Schilling, Phys. Rev. D 54 (1996) 2863.

[6] V. Bornyakov and M. Muller-Preussker, Nucl. Phys. Proc. Suppl. 106, 646 (2002).

[7] N. Sakumichi and H. Suganuma, Phys. Rev. D 90 (2014) no.11, 111501 doi:10.1103/PhysRevD.90.111501 [arXiv:1406.2215 [hep-lat]].

[8] M. N. Chernodub and M. I. Polikarpov, [arXiv:hep-th/9710205 [hep-th]].

[9] R. W. Haymaker, Phys. Rept. 315 (1999), 153-173 [arXiv:hep-lat/9809094 [hep-lat]].

[10] H. Shiba and T. Suzuki, Phys. Lett. B 333, 461 (1994).

[11] J. D. Stack, S. D. Neiman and R. J. Wensley, Phys. Rev. D 50, 3399 (1994).

[12] G. 't Hooft, in Proceedings of the EPS International, edited by A. Zichichi, p. 1225, 1976.

[13] S. Mandelstam, Phys. Rept. 23 (1976), 245-249

[14] O. Miyamura, Phys. Lett. B 353, 91 (1995).

[15] S. Kitahara, O. Miyamura, T. Okude, F. Shoji and T. Suzuki, Nucl. Phys. B 533, 576 (1998) 
[16] P. de Forcrand and M. D'Elia, Phys. Rev. Lett. 82, 4582 (1999) [arXiv:hep-lat/9901020].

[17] V. G. Bornyakov, M. I. Polikarpov, G. Schierholz, T. Suzuki and S. N. Syritsyn, Nucl. Phys. B Proc. Suppl. 153, 25-32 (2006) [arXiv:hep-lat/0512003 [hep-lat]].

[18] V. G. Bornyakov, I. Kudrov and R. N. Rogalyov, [arXiv:2101.04196 [hep-lat]].

[19] J. Smit and A. van der Sijs, Nucl. Phys. B 355, 603 (1991).

[20] M. Albanese et al. [APE Collaboration], Phys. Lett. B 192, 163 (1987).

[21] V. G. Bornyakov, V. V. Braguta, E. M. Ilgenfritz, A. Y. Kotov, A. V. Molochkov and A. A. Nikolaev, JHEP 03 (2018), 161 doi:10.1007/JHEP03(2018)161 [arXiv:1711.01869 [hep-lat]]. 